\title{
Toluene Detection in Aqueous Phase by Optical Fiber Sensors Integrated with Single-Walled Carbon Nanotubes
}

\author{
A. Crescitelli ${ }^{1}$, M. Consales ${ }^{1}$, M. Penza ${ }^{2}$, P. Aversa ${ }^{2}$, M. Giordano ${ }^{3}$ and A. Cusano ${ }^{1, *}$ \\ ${ }^{I}$ Optoelectronic Division-Engineering Department, University of Sannio, C.so Garibaldi 107, 82100 Benevento, Italy; \\ ${ }^{2}$ Department of Physical Technologies and New Materials, ENEA, SS. 7, Appia, km 714, 72100 Brindisi, Italy and \\ ${ }^{3}$ Institute for Composite and Biomedical Materials, CNR, Piazzale Enrico Fermi 1, 80055 Portici, Italy
}

\begin{abstract}
Recent preliminary studies have demonstrated the feasibility of using Single-Walled Carbon Nanotubes (SWCNTs) as sensitive coatings of optical fiber sensors for the detection of chemical traces in water at room temperature. A low- finesse extrinsic Fabry-Perot configuration was adopted as sensing scheme and Langmuir-Blodgett thin SWCNT films have been integrated to form the sensing optical cavity. This contribution reports on the improvement of the performance of such SWCNTs-based chemo-optic transducers based on a proper choice of the number of carbon nanotube monolayers. The results here shown reveal a strong enhancement in term of sensor sensitivity of more than one order of magnitude, combined with a significant reduction of the response times. The achieved sub-ppm resolution, the response rapidity, and the complete and fast recovery of the initial output signal corresponding to the condition of uncontaminated water reveal the potentiality of the improved version of the sensor to be applied for water monitoring applications.
\end{abstract}

Key Words: Optical fiber sensor, single-walled carbon nanotubes, toluene detection in water, LB deposition technique.

\section{INTRODUCTION}

Increasing environmental legislations, both in USA and $\mathrm{EU}$, dictate the controls of the release and of the concentration levels of many chemicals in the environment. Thus reliable monitoring of these substances in air and especially in water is required. In 2003, the Scientific Committee on Toxicity, Ecotoxicity and Environment indicates toluene among the main pollutants that should be monitored in drinking water. As a matter of fact toluene can cause many risks for the human health. Short-time toluene inhalation (of about $100 \mathrm{ppm}$ ) causes headaches, dizziness and eyes/nose irritation. Long-term exposure impairs the central nervous system, alters the blood count and causes other chronic effects. Toluene in water is hazardous firstly because its volatility is significantly released into the atmosphere and secondly because its relatively high solubility in water may give significant pollution problems for both surface water and groundwater. Therefore in many fields simple and reliable measurement devices able to detect such substance in water are demanded. Several analytical techniques can be used for this scope (and in general for water quality monitoring), including gas chromatography [1], UV spectroscopy [2] and also other special techniques based on immunoassay kits [3]. The principal negative aspects of these approaches is that they require repeated procedures of manual sample collection of the polluted water, resulting very expensive and timeconsuming. In addition the integrity of the samples could be compromised during their collection, transport and storage.

\footnotetext{
*Address correspondence to this author at the Optoelectronic DivisionEngineering Department, University of Sannio, C.so Garibaldi 107, 82100 Benevento, Italy; Tel: +390824305835; Fax: +390824305846;

E-mail:a.cusano@unisannio.it
}

To avoid these drawbacks, in the last decade, a strong effort has been put into the development of robust, inexpensive, on site and continuous analysis methods. Many research efforts have been devoted to optical fiber sensors due to their high versatility, sensitivity, robustness and capability to work in hard environmental condition [4]. Furthermore, these devices are potentially inexpensive and allow continuous, on site, remote measurements, without sampling procedure and, thus, with no sample contamination. Several fiber optic sensors for the detection of pollutants dissolved in water have been described, especially for hydrocarbons and pesticides. In particular, different evanescent wave based fiber-optic sensors applying polymer coated silver-halide $(\mathrm{AgX})$ fibers covered with thin hydrophobic polymer layers and coupled with FTIR spectrometers have been proposed during the past years [5-9]. They allowed qualitative and quantitative determination of a wide variety of organic analytes in the $\mathrm{mg} / \mathrm{l}$ to the low $\mu \mathrm{g} / \mathrm{l}$ concentration range. Limits of detection of 1 $\mathrm{ppb}$ and $0.1 \mathrm{ppm}$ for toluene in water were achieved respectively with an immunoassay-based fiber optic sensor [10] and with sensors employing polymeric membranes applied on several meters long segments of bent Polymer-Clad Silica (PCS) fibers $[11,12]$.

The use of straight segments of multimode fibers with inverted graded refractive index (IGI) profile in the core, coated by thin layer of polydimethylsiloxane polymer, was proposed by Chomat and co-workers, allowing toluene resolution limit of several ppm [13].

In addition, recently Skokankova and co-workers investigated the optical properties of xerogel layers applied on silica optical fibers and their sensitivity to toluene dissolved in water. By dip-coating the bare core of PCS fiber with layer 
prepared by sol based on tetraethoxisylane, methyltriethoxisylane and a mixture of methyltriethoxysilane and phenyltriethoxysilane, they reached resolution limits of a few ppm [14].

Large interest has thus been focused on the materials capable of improving the fiber optic sensor performance. At the same time, the discovery of carbon nanotubes (CNTs) [15] created much excitement and stimulated extensive research since their properties appeared promising for numerous possible applications. CNTs are nanostructured materials with unique structural, electronic, optical mechanical and thermal properties [16]. Their application fields include cold cathode flat panel display (FPD) [17], nanoelectronic devices $[18,19]$, nanorelays[20], super capacitors [21], tips for scanning probe microscopes [22], hydrogen storage [23, 24], quantum wires [25] and field effect transistors [26]. Their small size allows them to be used as extremely small layers to realize chemical sensors for volatile organic compounds (VOC), $\mathrm{H}_{2}, \mathrm{NO}_{2}$ and $\mathrm{NH}_{3}$ detection in gaseous environments $[27,28]$ and also strain sensors [29]. CNTs are also useful for biosensor improvement because of their capability to provide strong electrocatalytic activity and to minimize sensor surface fouling. Indeed such materials have already been used for the development of biosensors able to detect glucose [30-32] and organophosphorous compounds [33].

CNTs can be distinguished, according to their structural properties, into two groups: those consisting of a single wall of surface-arranged carbon atoms (SWCNTs) and those characterized by concentric multiwalls of surface-arranged carbon atoms (MWCNTs). Despite structural similarity to a single sheet of graphite (a semiconductor with zero band gap) SWCNTs behave as metallic or semiconducting material, depending on the way about which the graphite sheet is rolled to give the nanotube cylinder and appear more suitable than MWCNTs for sensor applications because of their higher gas adsorption capacity which gives higher gas sensitivities [34].

SWCNTs have already been exploited by the authors as highly sensitive layers for the development of high performance VOC silica optical fiber (SOF) sensors [35-37] and recently preliminary results demonstrated their potentiality to be exploited also for the detection of chemical pollutants in aqueous environment at room temperature [38]. In addition, the possibility to tailor the performance of these SWCNTsbased chemo-optic sensors, either in term of sensitivity and response rapidity, by means of a proper choice of the sensitive layer features has also been recently demonstrated [39].

In light of these considerations, this contribution reports on the improvement of the sensing performance of SWCNTsbased optoelectronic chemo-sensors for chemical trace detection in water environment. The results obtained evidenced that an opportune decrease of the number of SWCNT monolayers transferred upon the fiber tip promotes a sensitivity enhancement of more than one order of magnitude combined with a reduction from 20 minutes to 15 minutes of the mean response time. The data here reported reveal the potentiality of the proposed SWCNTs-based fiber optic chemo-sensors to successfully compete with the already proposed fiber optic transducers in the wide scenario of in-situ, real time, and low cost sensors for the monitoring of dissolved organic matters in water.

\section{METHODOLOGIES}

\section{Operating Principle and Interrogation Scheme}

The optoelectronic sensing configuration is essentially based on a low finesse and extrinsic Fabry-Perot (FP) interferometer and uses a thin sensitive SWCNT multilayer deposited at the distal end of a properly cut and prepared fiber. The key point of the proposed optoelectronic sensor is thus the dependence of the reflectance at the fiber/SWCNT film interface $\left(\mathrm{R}_{\text {Film }}\right)$ upon the optical and geometrical properties of the sensitive materials [40]. In particular, the adsorption of the target analyte molecules inside the carbon nanotube layer modulates its thickness and/or dielectric constant, leading to changes in the film reflectance and, thus, in the intensity of the optical signal reflected at the fiber/film interface. Among the variety of schemes proposed for the interrogation of a fiber optic sensor based on the reflectometric approach [41], here the attention has been focused on single wavelength continuous wave interrogation. This approach has the peculiarity of being simple to implement and requires just few widespread commercial and low-cost optoelectronic components while preserving excellent performance. In addition it enables the fabrication of cost-effective, reliable, robust and portable equipments, which are factors of crucial importance for in-situ and long-term monitoring applications and for the desired technology transfer to the market. The typical interrogation scheme enabling the single wavelength reflectance monitoring at the sensitive interface is shown in Fig. (1). The sensitive interface is lighted by a pigtailed Superluminescent Light Emitting Diode (SLED) operating in one of the two main telecommunication wavelengths, $1310 \mathrm{~nm}$. A source controller is used in order to keep the SLED at a constant temperature and feed it with an input current, which allows the source to emit an optical power of few $\mathrm{mW}$. This power is split by a $2 \times 2$ in-fiber directional coupler, providing the necessary connections between light source, sensing interface and two photoreceivers. The first photoreceiver is for the measurement of the signal reflected at the sensing interface $\left(\mathrm{V}_{\text {signal }}\right)$, while the second one is used to provide a valid power source intensity monitoring $\left(\mathrm{V}_{\text {source }}\right)$ enabling the compensation of the sensor response. The intensity compensation is obtained by considering the ratio between the voltage signals at the two photo-receivers. Synchronous detection is also typically implemented to enhance the system performance, by amplitude modulating the light source at $500 \mathrm{~Hz}$ and retrieving the photodetector voltages by using a dual channel lock-in amplifier.

\section{SWCNT Deposition Technique}

The realization of homogeneous thin films of SWCNTs with a controllable thickness is an important basis for the future development of their scientific understanding and technological applications. Proper manipulation techniques are required for applying thin-films of carbon nanotubes on substrates that do not allow direct grow methods. Although various proposals exist for their incorporation into devices [42], here the Langmuir-Blodgett (LB) technique has been chosen as way to transfer nanometer-scale thin layers of SWCNTs onto the distal end of standard optical fibers.

The LB is a well-known method for depositing defectfree, molecularly ordered ultra-thin organic films with a precise control over the architecture of the films at the molecu- 
lar level [43]. An additional advantage of this technique is that the monolayers can be deposited on almost any kind of solid substrate. However, these advantages have to be traded with the low speed of the deposition procedure (the films are deposited monolayer by monolayer) and the limited number of materials suitable for LB deposition.

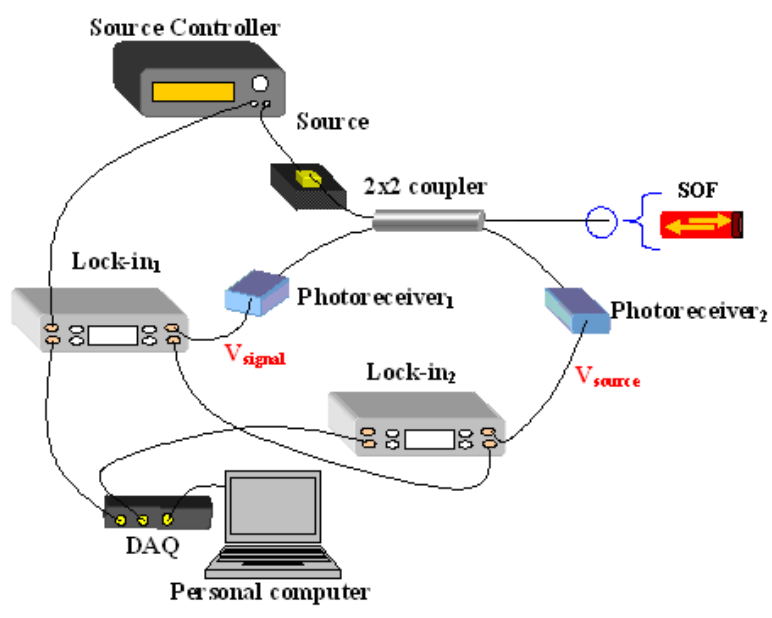

Fig. (1). Schematic illustration of the typical interrogation scheme adopted for the single wavelength reflectance monitoring of an optical cavity realized upon the fiber tip.

The SWCNT deposition procedure started by spreading a solution $(0.2 \mathrm{mg} / \mathrm{ml})$ of single-walled carbon nanotube pristine material (purchased from Carbon Nanotechnologies Inc., Houston, USA) in chloroform onto a sub-phase constituted by deionized water $(18 M \Omega)$ with $10^{-4} M$ of $\mathrm{CdCl}_{2}[36,37]$. The sub-phase $\mathrm{pH}$ and temperature were 6.0 and $23^{\circ} \mathrm{C}$, respectively. The monolayer has been compressed with a barrier rate of $15 \mathrm{~mm} / \mathrm{min}$ up to a surface pressure of $45 \mathrm{mN} / \mathrm{m}$. The single layer has been deposited onto the distal end of the SOF with a dipping rate of $3 \mathrm{~mm} / \mathrm{min}$ and the transfer ratio of the monolayer from the sub-phase to the substrate surface was in the range of 0.5-0.7. A standard single mode fiber was used, with a core and cladding diameter of approximately 9 and $125 \mu \mathrm{m}$, respectively. The fiber probes were prepared by stripping the optical fiber protective coating (1$2 \mathrm{~cm}$ length) from the fiber end. Then, they have been washed in chloroform and dried with gaseous nitrogen to be ready for the SWCNT deposition.

In the previous work, in which the authors presented preliminary results demonstrating the feasibility of SWCNTs to be applied for water quality monitoring applications, a sensitive overlay constituted of 12 SWCNT monolayers was used (approximately $24 \mathrm{~nm}$ ) [38]. Here, in light of the results of recent studies on the possibility of tailoring the sensing performance of carbon nanotubes-based chemo-optic sensors [39], a reduction of the number of carbon nanotube monolayers transferred on the end-face of the optical fiber was performed. In this case a sensitive coating made of only two monolayers (approximately $4 \mathrm{~nm}$ ) has been considered. This choice has been accomplished with the aim of minimizing the sensor response time. The toluene sensing performance of the two sensors, coated by 2 (sample A) and 12 (sample B) SWCNT monolayers, have been here compared.

\section{EXPERIMENTAL RESULTS}

The experimental set-up exploited for the investigation of the sensing features of SWCNT transducers in aqueous environment is shown in Fig. (2). The sensors have been settled in a proper Pyrex beaker containing pure water, inside which different volumes of toluene have been each time injected to simulate the condition of contaminated water at different concentrations. The polluted water has been continuously stirred to ensure the maximum dispersion of the analyte. The desorption aptitudes of the SWCNTs-based sensors have also been investigated by restoring, after each toluene injection, the initial condition of uncontaminated environment: to achieve this aim pure water was poured in the test beaker, while the contaminated water was simultaneously spilled out.

Before performing the toluene detection measurements in water, the stability of the fabricated SWCNT sensitive layers in an aqueous environment has been investigated.

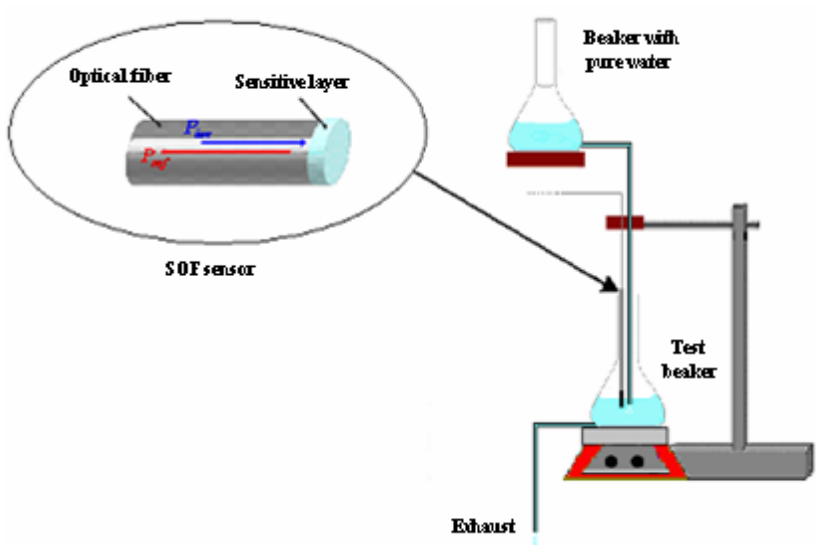

Fig. (2). Schematic view of the experimental set-up exploited for toluene detection measurements in water.

To achieve this aim, the carbon nanotubes-based SOF sensors have been inserted in the beaker containing pure water and left for several days inside it. In Fig. (3a and 3b) are reported the relative reflectance changes occurred for the two optoelectronic probes as a consequence of their insertion in water.

In both cases, as the optical probes were inserted in water, a strong and fast reflectance variation occurred as a consequence of the change in the external medium refractive index. In particular, as the external refractive index changes from air $(n \sim 1)$ to water $(n \sim 1.33)$, the reflectance at the filmexternal medium interface decreases leading to a significant diminution of the detected reflected signal.

This effect is followed by a slow variation of the reflectance itself (see the insets) until the equilibrium value is reached, which can be attributed to the interaction between sensitive layers and water molecules. This evidence suggests the occurring of a reorganization of the carbon nanotubes upon the sensor surface, leading to a modification of the overlay morphology. At the equilibrium, the ratio between the reflectance of sample $\mathrm{B}$ and that of sample A is approximately 5.6 (in air it was 1.8). As matter of fact, sample A exhibited a relative reflectance diminution of approximately 

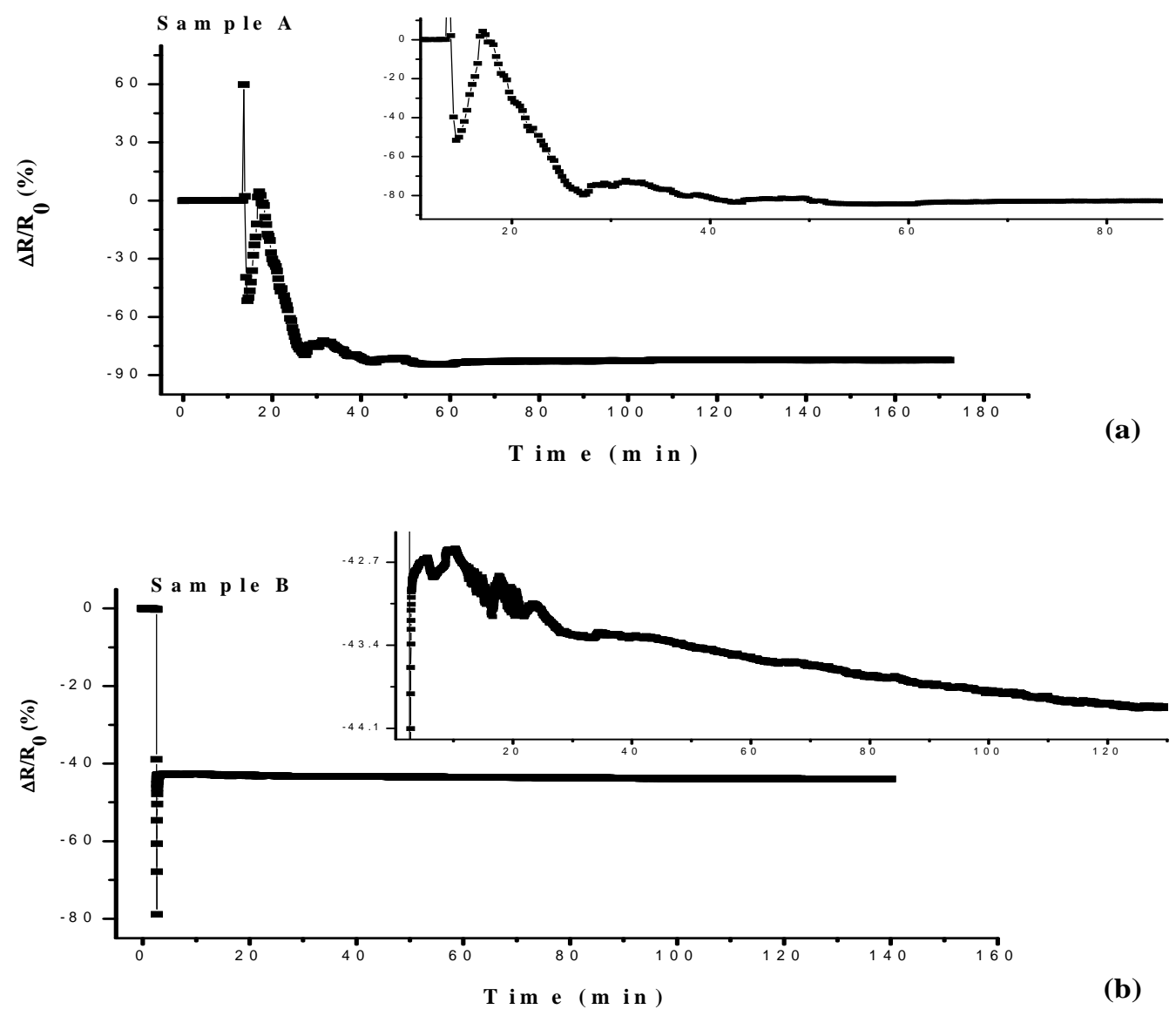

Fig. (3). Relative reflectance changes $\left(\Delta R / R_{0}\right)$ occurred to the two optoelectronic probes coated respectively by (a) 2 and 12 (b) monolayers of SWCNTs as a consequence of their insertion in water.

$82 \%$, almost doubled with respect to the one observed for sample B (approximately 44\%).

In any case it is evident that once reached the equilibrium, the reflectance signals are stable for both the sensors, demonstrating that no degradation of the SWCNT sensitive layers occurs. In fact a degradation of the fiber overlay would result in a modification of its optical or geometrical properties, thus leading to significant changes in the sensor output.

Successively, sample A and B have been tested for toluene detection in aqueous environment at room temperature. The toluene concentration has been chosen to be in the range 0-80 ppm $(\mu l / l)$ : different volumes of toluene were added to pure water in four subsequent injection to obtain concentration levels of 20,40, 60 and $80 \mathrm{ppm}$, respectively. The results obtained are shown in Fig. (4), where the normalized reflectance changes of both the samples versus time are reported. It is noteworthy that in the two cases, in correspondence of each toluene injection the interaction between the analyte molecules and the sensitive overlay results in the adsorption of such molecules within the SWCNT overlay.

In addition, as expected, the intensity of the sensor output changes increased with the toluene concentration. However it is clearly visible that the intensity of the reflectance variations observed for sample A are significantly greater than those of sample B. Complete and fast recoveries of the steady-state value of uncontaminated water were provided by the two carbon nanotubes-based SOF sensors, evidencing their excellent desorption capability and reusability. Also slight drifts in the sensor baseline can be noticed, which could be attributed to thermal changes since the test beaker is not perfectly thermo-stated. In particular, the temperature within the test beaker during experiments was approximately $24^{\circ} \mathrm{C}$ and the thermal drift was of approximately $3{ }^{\circ} \mathrm{C}$.

Further studies are currently under investigation to verify if also other causes could be responsible of the drift in the signal baseline. In order to compensate the effects of temperature variations on the sensors response a proper Fiber Bragg Grating, either included inside the test chamber or even integrated in the sensing optical fiber itself, can be used as temperature sensor [44].

Furthermore, the noise effect on the sensor performance has been estimated by considering the sensor output $(I)$ in the steady state levels corresponding to the absence of toluene within the test beaker. Taking into account the maximum scattering in the sensor level of the sample A over a time interval of about 10 minutes, standard deviations $\sigma_{N}$ of approximately $3.3 \cdot 10^{-4}$ from the mean value of the sensor output $I_{0}\left(I_{0}=0.2741\right)$ have been appreciated. By these results a minimum detectable change of the sensor output $\Delta I / I_{0 \min }$ (and thus a $\Delta R / R_{0 \min }$ ) of $1.2 \cdot 10^{-3}$ has been estimated for this sample. Similar calculation can be made for sample B, leading to a $\Delta R / R_{0 \min }$ of approximately $6 \cdot 10^{-4}[38]$. 


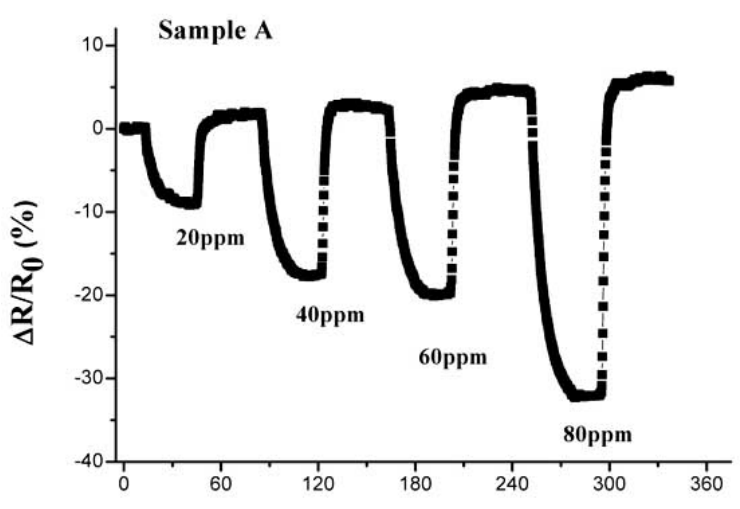

Time (min)

(a)

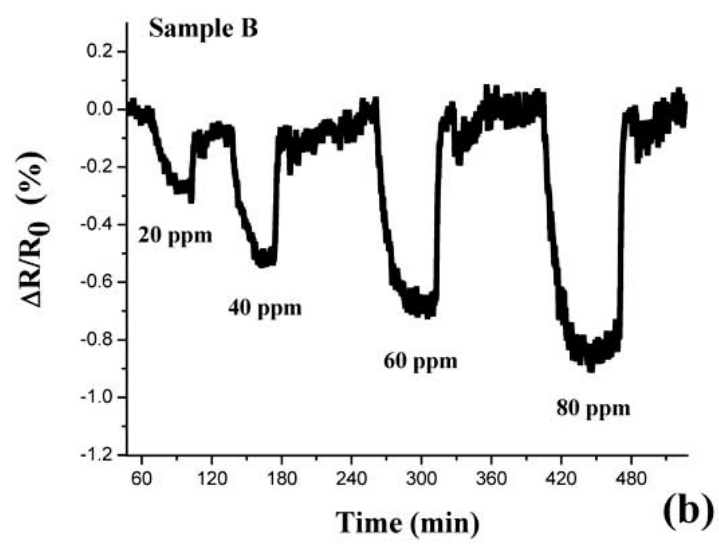

Fig. (4). Time responses of (a) sample A and (b) sample B on exposure to four different toluene concentrations, at room temperature.

\section{Sensitivity Analysis}

In this section, the results obtained from the comparison between the sensitivities against toluene in water for sample $\mathrm{A}$ and $\mathrm{B}$ are reported (the sensitivity is calculated as $\Delta R / R_{O} / C$, where $C$ is the analyte concentration). In particular, in Fig. (5) the sensor characteristic curves reporting the normalized reflectance changes versus the toluene concentrations are plotted for both the optoelectronic transducers.

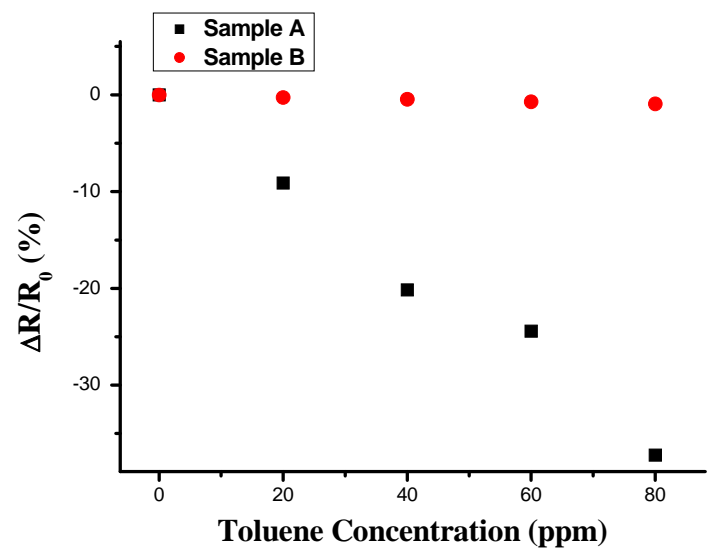

Fig. (5). Comparison between the calibration curves of sample A and $\mathrm{B}$ in water environment, at room temperature.
As it appears, high linearity has been obtained in both cases for all the toluene concentration range and a much higher sensitivity has been reached for the improved sensor coated by 2 monolayers of carbon nanotubes (approx. $4.5 \cdot 10^{-3} \mathrm{ppm}^{-1}$ ) than for the 12 SWCNT monolayers-based one (approx. $-1.2 \cdot 10^{-4} \mathrm{ppm}^{-1}$ ). In addition, taking into account the minimum detectable changes of the sensor output and the aforesaid sensitivities, resolutions of approx. $300 \mathrm{ppb}$ and 5 ppm have been estimated for sample A and B, respectively. These results clearly evidence the strong enhancement of more than one order of magnitude obtained in term of sensitivity by the use of sample A with respect to the counterpart sample B.

It is also interesting to make a comparison between the sensor performance against toluene in aqueous and gaseous environment. To achieve this aim, the room temperature toluene sensitivities of sample A and B in the two environments have been summarized in Table $\mathbf{1}$ (the sensitivity values in case of air chemical detection measurements are reported in previous works [37-39]).

The values reported in Table $\mathbf{1}$ show that very different responses can be obtained depending on the environment in which the adsorption measurements are carried out.

Table 1. Toluene Sensitivities of Sample A and B in Air and Water Environment

\begin{tabular}{|c|c|c|}
\hline \multirow{2}{*}{ ENVIRONMENT } & \multicolumn{2}{|c|}{ SENSITIVITY $\left(\mathrm{ppm}^{-1}\right)$} \\
\cline { 2 - 3 } & Sample A & Sample B \\
\hline \hline AIR & $2 \cdot 10^{-4}$ & $-1.5 \cdot 10^{-4}$ \\
\hline WATER & $-4.5 \cdot 10^{-3}$ & $-1.2 \cdot 10^{-4}$ \\
\hline
\end{tabular}

Such differences can be attributed to the dependence of the reflectance on the surrounding refractive index combined to different adsorption characteristics occurring in the two environments. This explains also the key role played by the thickness of the adsorbent material. As matter of fact, a toluene sensitivity which is positive and more than one order of magnitude lower than the one obtained in water has been obtained for sample A in case of toluene vapor detection in air $\left(2.0 \cdot 10^{-4} \mathrm{ppm}^{-1}\right)$. On the contrary, the performance of Sample B in the two different environments resulted quite similar, with the sensitivity in air slightly higher $\left(-1.5 \cdot 10^{-4}\right.$ $\mathrm{ppm}^{-1}$ ) than the one observed for the toluene trace detection in water. This evidences once more that in order to significantly improve the performance of the SWCNTs-based optochemical sensor for a specific environmental monitoring application, a proper choice of the sensing layer features is strictly required.

\section{Dynamic Characteristics}

In this last paragraph the dynamical characteristics of the two tested SWCNTs-based sensors in water environment at room temperature have also been investigated. In particular, the response (recovery) time has been calculated as the time needed to pass from $10 \%$ to $90 \%(90 \%-10 \%)$ of the total signal shift occurring on toluene adsorption (desorption). 
The results shown in Fig. (6) indicate that the two tested probes are characterized by almost similar recovery times (4 minutes for both) and quite different response times (15 and 20 minutes respectively for sample A and B). This means that a reduction of five minutes in the rapidity of the response has been obtained by the use of the improved sensor. However this aspect should be better investigated and is currently under study. In particular, an automated chamber for the liquid handling is being used in order to assess the influence of the experimental setup exploited for the adsorption measurements on the time characteristics of the two sensors. This will be useful also for a deeper understanding of the interaction dynamics between sensitive layer and analyte molecules.

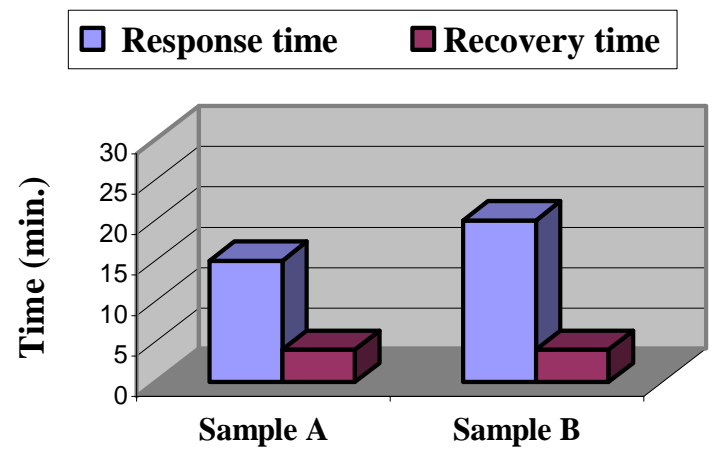

Fig. (6). Comparison between the mean response and recovery times in aqueous environment of sample A and B.

\section{CONCLUSION}

In conclusion in this work, the sensing capabilities of SWCNTs-based SOF sensors for the detection of chemical traces in water environment, at room temperature, have been improved by a proper choice of the sensitive overlay features. The proposed opto-chemical sensors have been arranged in a Fabry-Perot type reflectometric configuration and the Langmuir-Blodgett technique has been used to fabricate the two samples characterized by a different number of carbon nanotube monolayers. Before the analyte detection measurements, the stability of both the sensors in aqueous environment has been proved, demonstrating that no degradations of the SWCNT layer occurred. The experimental measurements have been carried out by exposing the two fabricated samples to different concentrations of toluene (in the range $0-80 \mathrm{ppm}$ ) in water and at room temperature. The analyte concentration within the test ambient has been continuously monitored through the changes occurring on exposure in the relative reflectance at the optical fiber-sensitive film interface. The results reported here demonstrate the strong sensitivity enhancement of the improved version of the chemo-optic sensor of more than one order of magnitude maintaining the linear operation. The new obtained resolution of $300 \mathrm{ppb}$, the response rapidity as well as the fast and complete recovery of the steady state value of uncontaminated water evidence the strong potentiality of the SWCNTsbased opto-chemical probes to be successfully applied to water quality monitoring applications. Finally, strong differences have been found in the sensor responses in case of air or water chemical monitoring, proving that in order to sig- nificantly improve the performance of the proposed optoelectronic sensors for a specific environmental monitoring application, a proper choice of the sensing layer features is strictly required. However, further experimental tests are currently in progress to investigate the selectivity of the proposed sensing device, which is expected to be quite weak. The low sensor ability to discriminate among different analytes is still one of the major concerns in chemical sensing. Two approaches are typically followed to solve this issue: the first one relies on the sensitive layer functionalization in order to achieve a higher affinity of the material towards specific chemical species [45]; the second one is based on the use of a hybrid system composed of multiple transducers coated by different sensitive materials in the form of an array. Here, sensors with a poor selective response, when considered collectively, provide unique patterns typical for each analyte. The generated response patterns are interpreted by pattern-recognition algorithm for the selective detection [46]. This approach has already been investigated and exploited by the authors for the VOC detection in air [36], and is currently under investigation.

\section{REFERENCES}

[1] Djozan D and Assadi Y. Monitoring of polycyclic aromatic hydrocarbons in water using headspace solid-phase microextraction and capillary gas chromatography. Microchem J 1999; 63 (2): 276.

[2] Vogt F, Tacke M, Jakusch M, Mizaikoff B. A UV spectroscopic method for monitoring aromatic hydrocarbons dissolved in water. Anal Chim Acta 2000; 422 (2): 187.

[3] Pichon V, Bouzige M, Hennion MC. New trends in environmental trace-analysis of organic pollutants: class-selective immunoextraction and clean-up in one step using immunosorbents. Anal Chim Acta 1998; 376 (1): 21.

[4] Lee B, Review of the present status of optical fiber sensors. Opt Fiber Technol 2003; 9: 57-79.

[5] Krska R, Taga K, Kellner R. New IR fiber-optic chemical sensor for in-situ measurements of chlorinated hydrocarbons in water. Appl Spectrosc 1993; 47(9): 1484-87.

[6] Mizaikoff B, Goebel R, Krska R, et al. Infrared fiber-optical chemical sensors with reactive surface coatings. Sensors and Actuators B Chem 1995; 29(1-3): 58-63.

[7] Walsh JE, MacCraith BD, Meaney M, et al. Sensing of chlorinated hydrocarbons and pesticides in water using polymer coated midinfrared optical fibers. Analyst 1996; 121(6): 789-92.

[8] Jakusch M, Mizaikoff B, Kellner R, Katzir A. Towards a remote IR fiber-optic sensor system for the determination of chlorinated hydrocarbons in water. Sens. and Actuators B Chem 1997; 38(1-3): 83-87.

[9] Regan F, MacCraith BD, Walsh JE, O'Dwyer K, Vos JG, Meaney M. Novel Teflon-coated optical fibers for TCE determination using FTIR spectroscopy. Vib Spectrosc 1997; 14(2): 239-46.

[10] Ives JT, Doss HM, Sullivan BJ, Stires JC, Bechtel JH. Fiber optic immunosensors to monitor small-molecule analytes in groundwater. R.A. Lieberman (Ed.), Proceedings of the SPIE 3540 Chemical, Biochemical and Environmental Fiber Sensors X Boston, USA, Nov 1999; 2: 36-44.

[11] Burck J, Mensch M, Kramer K. Field experiments with a portable fiber-optic sensor for monitoring hydrocarbons in water. Field Anal Chem Technol 1998; 2 (4): 205-19.

[12] Burck J, Schlagenhof M, Roth S, Mathieu H. Kinetic evaluation method for SPME-NIR measurements of analytes with long equilibration time. Field Anal Chem Technol 2001; 5 (3): 131-42.

[13] Chomat M, Berkova D, Matejec V, et al. Optical detection of toluene in water by using IGI fibers. Mater Sci Eng C 2002; 21: 211- 5.

[14] Skokankova J, Mrazek J, Matejec V, et al. Properties of xeroge layers for the detection of toluene in water. Mater Sci Eng C 2006; 26: $208-13$.

[15] Iijima S. Helical microtubules of graphitic carbon Nature (London) 1991; 354: 56-58. 
[16] Dresselhaus MS, Dresselhaus G, Avouris Ph. Carbon Nanotubes: Synthesis, Structure, Properties, and Applications, Berlin: Springer, 2001.

[17] Choi WB, Chung DS, Kang JH, et al. Fully sealed, high-brightness carbon-nanotube field emission display. Appl Phys Lett 1999; 75: 3129.

[18] Collins PG, Zetti A, Bando H, Thess A, Smalley RE. Nanotube Nanodevice. Science 1997; 278: 100-3.

[19] Lefebre J, Lynch J.F, Llaguno M, Radosavljevic R, Johnson AT. Single-wall carbon nanotube circuits assembled with an atomic force microscope. Appl Phys Lett 1999; 75: 3014-6.

[20] Kinaret JM, Nord T, Viefers S. A carbon-nanotube-based nanorelay. Appl Phys Lett 2003; 8: 1287-9.

[21] Niu C, Sichel EK, Hoch R, Moy D, Tennent H. High power electrochemical capacitors based on carbon nanotubes electrodes. Appl Phys Lett 1997; 70: 480-2.

[22] Dai H, Hafner JH, Rinzler AG, Colbert DT, Smalley R. Nanotubes as nanoprobes in scanning probe microscopy. Nature 1996; 384 : 147-50.

[23] Bauschlicher CWJr, So CR. High Coverages of Hydrogen on $(10,0),(9,0)$ and $(5,5)$ Carbon Nanotubes. Nano Lett 2002; 2: 337 41 .

[24] Ye Y, Ahn CC, Witham C, et al. Hydrogen adsorption and cohesive energy of single-walled carbon nanotubes. Appl Phys Lett 1999; 74: 2307-9.

[25] Tans SJ, Devoret MH, Dai H, et al. Individual single-walled carbon nanotubes as quantum wires. Nature 1997; 386: 474-6.

[26] Bradley K, Gabriel JCP, Star A, Gruner G. Short-channel effects in contact-passivated nanotube chemical sensors, Appl Phys Lett 2003; 83: 3821-3.

[27] Someya T, Small J, Kim P, Nuckolls C, Yardley JT. Alcohol vapor sensors based on single walled carbon nanotube field effect transistors. Nano Lett 2003; 3: 877-81.

[28] Wong YM, Kang WP, Davidson JL, Wisitsora A, Soh KL. A novel microelectronic gas sensor utilizing carbon nanotubes for hydrogen gas detection. Sens Actuators B Chem 2003; 93: 327-32.

[29] Kang I, Schulz MJ, Kim JH, Shanov V, Shi D. A carbon nanotube strain sensor for structural health monitoring. Smart Material and Structures 2006; 15: 737-48.

[30] Wang J, Musameh M, Lin Y. Solubilization of carbon nanotubes by Nafion toward the preparation of amperometric biosensors. J Am Chem Soc 2003; 125: 2408-9.

[31] Wang J, Musameh M. Carbon nanotube/Teflon composite electrochemical sensors and biosensors. Anal Chem 2003; 75: 2075-9.
[32] Lin Y, Lu F, Tu Y, Ren ZF. Glucose Biosensors Based on Carbon Nanotube Nanoelectrode Ensembles. Nano Lett 2004; 4: 191-5.

[33] Lin Y, Lu F, Wang J. Disposable carbon nanotube modified screenprinted biosensor for amperometric detection of organophosphorous pesticides and nerve agents. Electroanalysis 2004; 16: 145-9.

[34] Chopra S, Pham A, Gaillard J, Parker A, Rao AM. Carbonnanotube-based resonant-circuit sensor for ammonia. Appl Phys Lett 2002; 80: 4632-4.

[35] Penza M, Cassano G, Aversa P, et al. Alcohol detection using carbon nanotubes acoustic and optical sensors. Appl Phys Lett 2004; 85: 2378-81.

[36] Penza M, Cassano G, Aversa P, et al. Carbon nanotube acoustic and optical sensors for volatile organic compound detection Nanotechnology 2005, 16: 2536-47.

[37] Consales M, Campopiano S, Cutolo A, et al. Carbon nanotubes thin films fiber optic and acoustic VOCs sensors: Performances analysis. Sens Actuators B Chem 2006; 118: 232-42.

[38] Consales M, Crescitelli A, Campopiano S, et al. Chemical Detection in Water by Single-Walled Carbon Nanotubes-based Optical Fiber Sensors, IEEE Sensors Lett 2007; 7: 1004-5.

[39] Consales M, Cutolo A, Penza M, et al. Carbon Nanotubes Coated Acoustic and Optical VOCs Sensors: Towards the Tailoring of the Sensing Performances. IEEE Transactions on Nanotechnology; in press.

[40] Macleod HA. Thin-film optical filters. Institute of Physics Publishing Bristol and Philadelphia 2001.

[41] Kersey AD, Dandrige A. Applications of fiber-optic sensors. IEEE Transactions on components, hybrid and manufacturing technology 1990; 13: 137-43.

[42] Bachtold A, Hadley P, Nakanishi T, Dekker C. Logic circuits with carbon nanotube transistors. Science 2001, 294: 1317-20.

[43] Petty MC. Langmuir-Blodgett Films: An Introduction. Cambridge: Cambridge University Press 1996.

[44] Cusano A, Persiano GV, Russo M, Giordano M. Novel optoelectronic sensing system for thin polymer films glass transition investigation. IEEE Sens J 2004; 4: 837-44.

[45] Balasubramanian K, Burghard M. Chemically functionalized carbon nanotubes. Small 2005; 1: 180-92

[46] Zaromb S, Stellar JR. Theoretical basis for identification and measurement of air contaminants using an array of sensors having partially overlapping sensitivities. Sens Actuators B Chem 1984; 6: $225-43$.

(c) Crescitelli et al.; Licensee Bentham Open.

This is an open access article distributed under the terms of the Creative Commons Attribution License (http://creativecommons.org/licenses/by/2.5/), which permits unrestrictive use, distribution, and reproduction in any medium, provided the original work is properly cited. 